\title{
Evaluación de un Programa para Fortalecer a los Cuidadores Familiares de Enfermos Crónicos
}

\author{
Lucy Barrera-Ortíz ${ }^{1}$, Natividad Pinto-Afanador ${ }^{2}$ y Beatriz Sánchez-Herrera ${ }^{3}$ \\ ${ }^{1}$ Enfermera. Especialista en enfermería pediátrica, M. Sc. en enfermería. Departamento de cuidado y \\ práctica de enfermería, Facultad de Enfermería, Universidad Nacional de Colombia. E-mail: \\ lbarrerao@unal.edu.co \\ ${ }^{2}$ Enfermera. Especialista en enfermería neurológica, M. Sc. en Enfermería. Departamento de cuidado y \\ práctica de enfermería, Facultad de Enfermería, Universidad Nacional de Colombia. E-mail: \\ rnpintoa@unal.edu.co \\ 3 Enfermera. M. Sc. Enfermería. Departamento de cuidado y práctica de enfermería, Facultad de \\ Enfermería, Universidad Nacional de Colombia. E-mail: cbsanchezh@unal.edu.co
}

Recibido 15 Noviembre 2005/Enviado para Modificación 2 Abril 2006/Aceptado 29 Mayo 2006

\section{RESUMEN}

Objetivo El estudio evaluó la efectividad del programa "Cuidando a los cuidadores", diseñado para fortalecer la habilidad de cuidado de los cuidadores familiares de personas con enfermedad crónica.

Métodos Se utilizó un abordaje cuantitativo de tipo cuasiexperimental con grupos de estudio y control y medición de la habilidad de cuidado antes y después de la intervención. Se incluyeron en el estudio 250 cuidadores familiares.

Resultados Los resultados incluyen la descripción de cada uno de los grupos de cuidadores estudiados en términos de sexo, edad, relación familiar, tiempo de cuidado y grado de dependencia de la persona cuidada. Presentan los niveles de habilidad de cuidado inicial y final de cada grupo y la comparación entre ellos. Se encontraron en los cuidadores similitudes de género y responsabilidad de cuidar a personas más jóvenes y diferencias en la procedencia, la funcionalidad de las personas cuidadas, la experiencia acumulada y la edad. La habilidad de cuidado fue homogénea en los grupos antes de la intervención, pero diferente en su totalidad y el componente paciencia al finalizar el programa.

Discusión Los hallazgos ratifican lo señalado en la literatura sobre distribución de género, edad, asistencia en el hogar y baja habilidad de los cuidadores para responder a la tarea de cuidado de un familiar enfermo crónico. Los resultados permiten sugerir que el programa "Cuidando a los cuidadores" apoya el incremento de habilidades de cuidado y aporta con ello una opción 
en el campo de la cronicidad que debe ser más explorada para el ámbito de América Latina.

Palabras Clave: Cuidadores, familia, enfermedad crónica (fuente: $\mathrm{MeSH}$, BIREME).

\section{ABSTRACT \\ Evaluating a programme for strengthening family-caregivers' ability to care for the chronically ill}

Objective The study evaluated the effectiveness of the "Caring for the caregivers" programme which was designed to strength family caregivers' ability to care for people suffering from chronic disease.

Methodology The study used a quasi-experimental design, having study and control groups, and measured caring ability before and after the intervention. 250 family caregivers were included in the study.

Results The results included a description of the caregiver groups in terms of gender, age, family relationship, caring time and care receiver's dependence level. They also included initial and final levels regarding caring ability in each group, as well as comparing them to each other. Similarities were found in gender and responsibility for caring for younger people; differences were found in the service of origin, care-receiver functionality, accumulated experience and age. Caring ability was homogeneous in groups before the intervention but different in total level and patience following the programme.

Discussion The findings agreed with the literature in terms of gender distribution, age, home assistance and caregivers' low levels of ability in terms of taking care of a family member suffering from chronic disease. The results suggested that the "Caring for the caregivers" programme has helped to increase caregivers' caring abilities and in doing so provided an option regarding the chronically ill which must be further explored within a LatinAmerican setting.

Key Words: Family-caregiver, chronically ill (source: DeCS, NLM).

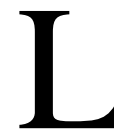
a sociedad vive el reto de responder al cuidado de la salud en situaciones de enfermedad crónica no transmisible que se han convertido en un problema progresivo y prioritario (1). Las implicaciones, las necesidades asistenciales y los retos que ocasionan estas enfermedades han sido ampliamente documentados (2-5).

Según los reportes, en el caso de la cultura latina el cuidador familiar de una persona con enfermedad crónica se comporta diferente frente a sus compromisos de cuidado, ve afectada su calidad de vida en especial su salud, ve 
comprometido su núcleo familiar y por lo general cuenta con soporte social adecuado (6-10).

Son múltiples las propuestas para intervenir con estos cuidadores familiares pero muy pocas las que se han adelantado en el contexto regional y menos aún aquellas que han sido evaluadas con rigor (11-12). Con base en la documentación de la falta de habilidad que los cuidadores tienen para responder a sus tareas de cuidado, de los beneficios del apoyo que brindan los diversos programas para los cuidadores y de la necesidad de generar y evaluar nuevas intervenciones con muestras más amplias, grupos de control más significativos, análisis de costos, diseños rigurosos e inclusión de estrategias y condiciones no exploradas, surge esta nueva propuesta (13-29).

El objetivo del estudio fue evaluar la efectividad del programa "Cuidando a los cuidadores", un programa desarrollado para fortalecer la habilidad de cuidado de los cuidadores familiares de personas con enfermedad crónica.

Este estudio retoma planteamientos conceptuales de Nkongho y Newman Los primeros, señalan que la habilidad de cuidado es el potencial de cuidado que tiene la persona adulta que incluye una dimensión cognoscitiva y una actitudinal identificadas y medidas con indicadores de conocimiento, valor y paciencia; la habilidad vista así puede evaluarse a través de la comunicación directa con cada persona. Fortalecer la habilidad de cuidado facilita la responsabilidad del cuidador (29). Los segundos, establecen la salud como una totalidad que incluye expresiones de enfermedad y de no enfermedad y aceptan que el individuo se expresa a través de un patrón (30-31). Las personas que viven con enfermedad crónica y sus cuidadores tienen sus propios patrones y reconocerlos es una forma de crecer que se relaciona con la capacidad de cuidado.

\section{MATERIALES Y MÉTODOS}

El estudio se realizó en Bogotá entre los años 2004 y 2005 con 250 cuidadores familiares de personas con enfermedad crónica. El diseño fue cuasiexperimental, dividiendo el total de cuidadores en dos grupos, uno de estudio y otro de control. Para respetar su agrupación natural no se asignaron los cuidadores a los grupos en forma aleatoria.

Los sujetos del estudio eran adultos que cumplían funciones de cuidado de un familiar con enfermedad crónica y fueron remitidos a la Facultad de Enfermería de la Universidad Nacional de Colombia desde diversas institu- 
ciones de salud del país, para ser atendidos de manera grupal o individual. Conformaron el grupo de estudio 125 de estos cuidadores vinculados al programa "Cuidando a los cuidadores". El grupo control se constituyó con otros 125 cuidadores familiares de características similares que no fueron vinculados a ese programa.

La medición inicial y final de la habilidad del cuidador familiar para cuidar se realizó con el inventario de habilidad de cuidado, CAI ${ }^{\circledR}$, (por sus siglas en inglés). Este instrumento tiene 37 ítems con graduación de tipo Likert y se compone de tres subescalas: conocimiento, valor y paciencia (29). $\mathrm{Su}$ validez es de 0.80 y su confiabilidad de 0.84 . Esta confiabilidad se ratificó con población colombiana. Se incluyeron además preguntas generales para caracterizar a los cuidadores y se utilizó el perfil PULSES para hacer la valoración funcional del receptor. Este perfil permite cuantificar el grado de dependencia de una persona y ha sido empleado con éxito como herramienta de valoración clínica en situaciones de cronicidad (32).

La intervención sobre el grupo de estudio fue el programa "Cuidando a los cuidadores" desarrollado con base en la evidencia disponible para fortalecer su habilidad de cuidar. El mismo tiene una duración de 10 semanas con una intensidad de 3 horas semanales y atiende de forma simultánea un promedio de 20 cuidadores familiares. El programa está organizado con un material audiovisual y escrito que lo acompaña y cuenta con guías estructuradas en tres módulos de acuerdo con los componentes de la habilidad de cuidado.

La intervención del grupo control se hizo preguntando a los usuarios qué tópico o actividad de su interés relacionada con su rol de cuidadores querían tratar. Este grupo no recibió un material educativo específico. Los encuentros con este grupo fueron en promedio 10 por grupo durante los doce meses del estudio.

El escenario de la intervención terapéutica dependió en ambos grupos de la necesidad y ubicación de los cuidadores. Las variables de confusión se controlaron al tomar grupos muy similares en todas sus características, y dar a un grupo el programa "Cuidando a los cuidadores" y al otro una intervención convencional.

El estudio consideró los aspectos éticos referentes al costo beneficio, el consentimiento informado la participación voluntaria y manejo confidencial de la información. 
La comparación de los dos grupos estudiados se realizó en cada tiempo (inicial y final) utilizando la estadística t de student con chequeo previo del supuesto de homogeneidad de varianzas. Por otra parte, cada grupo fue comparado antes y después de la intervención por medio de la estadística tpareada. Los datos obtenidos a través de los cuestionarios iniciales y finales se incorporaron a una matriz creada en Microsoft Excel y se procesaron en el software estadístico MINITAB versión 14.0, con el cual se generaron estadísticas descriptivas e inferenciales. Las hipótesis planteadas fueron verificadas a un nivel de confianza del $95 \%$.

\section{RESULTADOS}

\section{Características de los cuidadores}

En el grupo de estudio, de 125 cuidadores, el 80,8 \% de las personas asistían a servicios ambulatorios o se encontraban en el hogar y el 19,2 \% al servicio de Hospitalización. El 18,4 \% de los cuidadores eran hombres y un 81,6 \% corresponde a mujeres. La edad del cuidador estaba entre los 18 y 35 años en el 35,2 \% de los casos, entre 36 y 59 años el 45,6 \% y eran mayores de 60 en $19,2 \%$ de los casos. La edad del paciente era superior al rango de la edad de su cuidador en el 35,2 \% de los casos, en el mismo rango de la edad del cuidador en el 1,6 \% y de edad inferior al rango del cuidador en el 63,2 \% de los casos. El 14,6 \% de los cuidadores tenían una experiencia de 0 a 6 meses, el 16,8 \% de 7 a 18 meses, 16,0 \% de 19 a 36 meses y 52,8 \% tenían mas de 37 meses de experiencia en el cuidado. El nivel de funcionalidad medido a través del perfil PULSES reportó que tenían un bajo nivel de dependencia el 68,8 \% de los casos, un nivel medio el 6,4 \% y un alto nivel de dependencia el $24,8 \%$ de los casos.

En el grupo control con el mismo número de 125 cuidadores, se encontró que el 47,9 \% de las personas asistían a servicios ambulatorios o se encontraban en el hogar y el 52,1 \% al servicio de hospitalización. El 19,1 \% de los cuidadores eran hombres y un 80,9 \% corresponde a mujeres. La edad del cuidador estaba entre los 18 y 35 años en el 46,8 \% de los casos, entre 36 y 59 años el 27,7 \% y eran mayores de 60 el 25,5\% de los casos. La edad del paciente era superior al rango de la edad de su cuidador en el 39,4\% de los casos, en el mismo rango de la edad del cuidador en el 17,0 \% y de edad inferior al rango del cuidador en el 43,6 \% de los casos. El 44,7 \% de los cuidadores tenían una experiencia de 0 a 6 meses, el 20,2 \% de 7 a 18 meses, $12,8 \%$ de 19 a 36 meses y 22,3 \% tenían mas de 37 meses de experiencia en el cuidado. El nivel de funcionalidad medido a través del perfil PULSES re- 
portó que tenían un bajo nivel de dependencia el 28,0 \% de los casos, un nivel medio el 23,4 \% y un alto nivel de dependencia el 48,8 \% de los casos.

Habilidad de cuidado de los cuidadores

La habilidad de cuidado de los cuidadores fue analizada de manera total y por componentes. En el primer análisis (CAI total), se encontró que en el grupo de estudio y en el control incrementaron su valor promedio de la medición inicial a la final (Tabla No.1).

En el análisis por componentes se encontró, en la dimensión valor, cuyos resultados podrían encontrarse entre 13 y 91 que el grupo de estudio presentó una mediana de 50 en su medición inicial con una DS de 13,5 y una mediana 55,3 con una DS de 14,7 en su medición final. El grupo control pasó de una mediana de 49,8 a 53,1 con DS de 14,7 en la medición inicial a 11,3 en la final.

Tabla 1. Habilidad de cuidado según momentos de medición

\begin{tabular}{ccccccc}
\hline Grupo & $\begin{array}{c}\text { Momento } \\
\text { de la } \\
\text { Medición }\end{array}$ & Media & $\begin{array}{c}\text { Desviación } \\
\text { Estándar }\end{array}$ & $\begin{array}{c}\text { Valor } \\
\text { mínimo }\end{array}$ & Mediana & $\begin{array}{c}\text { Valor } \\
\text { máximo }\end{array}$ \\
\hline Control & Inicial & 189,1 & 23,3 & 77,0 & 190,5 & 232 \\
\hline \multirow{2}{*}{ De estudio } & Final & 191,1 & 18,2 & 136,0 & 191,0 & 230 \\
\hline & Inicial & 191,2 & 19,2 & 91,0 & 190,0 & 239 \\
\hline
\end{tabular}

En el componente conocimiento cuyos resultados podían oscilar entre 14 y 98 se encontró que la mediana en el grupo de estudio pasó de 81,3 en su medición inicial con una DS de 9,9 a 82,3 con una DS de 10,6 en su medición final. El grupo control pasó de 79,6 en su medición inicial con una DS de 11,5 a una medición final de 80 con una DS de 8,8.

En el componente paciencia cuyos posibles valores se encontraban entre 10 y 70 se encontró que en el grupo de estudio pasó de 60 con una DS de 6,7 en su medición inicial a 61,1 con una DS de 7,5 en su medición final. El grupo control pasó de 59,7 con una DS de 8,3 en su medición inicial a 58 con una DS de 6,5 en su medición final.

Comparación de los grupos de estudio y control

Los grupos de estudio y control fueron comparados en cada momento (antes y después de la intervención) utilizando la prueba t de Student, considerando la característica aditiva de la escala utilizada en la que cada individuo ob- 
tiene un valor cuantitativo, y previo chequeo de normalidad y homogeneidad de varianzas en el grupo de datos. Esta prueba permitió contrastar la hipótesis entre las medias de los grupos. En todas las dimensiones los grupos resultaron ser similares en el momento inicial del estudio y en CAI total y el componente paciencia los grupos resultaron ser diferentes en el momento final del estudio (Tabla 2).

Al realizar la comparación del momento inicial vs. momento final en el CAI total y los componentes en cada grupo de estudio, se encontró en el grupo estudio que todas las categorías incrementaron su nivel, de las cuales el CAI total y el componente valor fueron estadísticamente significativas $\mathrm{P}<0.05$. En el grupo control, el componente paciencia disminuyó su nivel y el componente valor incrementó significativamente $\mathrm{p}<0.05$ (Tabla 3).

Tabla 2. Comparación entre los grupos de estudio y control, antes y después de la intervención

\begin{tabular}{lcc}
\hline \multicolumn{1}{c}{ Item/Medición } & Inicial & Final \\
\hline CAl Total & $p=0,452$ & $p=0,007$ \\
\hline Valor & $p=0,936$ & $p=0,231$ \\
\hline Conocimiento & $p=0,237$ & $p=0,070$ \\
\hline Paciencia & $p=0,772$ & $p=0,001$ \\
\hline
\end{tabular}

Tabla 3. Comparación de los momentos inicial y final entre los grupos

\begin{tabular}{ccc} 
& de estudio y control & \\
\hline Categoría/grupo & Grupo Estudio & $\begin{array}{c}\text { Grupo } \\
\text { Control }\end{array}$ \\
\hline CAl total & $p=0.011$ & $p=0.371$ \\
Valor & $p=0.005$ & $p=0.042$ \\
Conocimiento & $p=0.446$ & $p=0.719$ \\
Paciencia & $p=0.262$ & $p=0.029$ \\
\hline
\end{tabular}

\section{DISCUSIÓN}

Los grupos de cuidadores resultaron ser casi iguales en su distribución de género. Por el contrario se notan diferencias en el servicio en el cual fueron contactados y en el nivel de funcionalidad. La coincidencia entre el servicio de hospitalización y el bajo nivel de funcionalidad es llamativa. La distribución por edades señala que hay mayoría de cuidadores en edades entre los 
36 a 59 en el grupo de estudio mientras que en el control el grupo mayoritario se encuentra entre los 18 y 35 años. Esto puede deberse a que en la muestra del segundo grupo se incluyeron los cuidadores de una institución pediátrica.

Llama la atención que en ambos grupos haya más cuidadores cuidando a personas en un rango de edad más joven que ellos. A pesar de coincidir con el perfil epidemiológico nacional que genera enfermedad por trauma y violencia, no deja de resultar preocupante el futuro de estos receptores del cuidado y sus familiares mayores que difícilmente contarán con el apoyo de la generación joven para su propio cuidado en años venideros.

En el grupo de estudio hay una mayor proporción de cuidadores con más de 6 meses de cuidado. Este hallazgo puede asociarse a la ubicación de los grupos en instituciones hospitalarias donde las personas manejan situaciones más agudas dentro del proceso de cronicidad. Podría pensarse que algunas de esas situaciones pudieran asociarse con la falta de experiencia de los cuidadores. El nivel de funcionalidad medido con el perfil PULSES refleja que en el grupo de estudio la funcionalidad del receptor del cuidado es mayor, lo cual coincide como el hallazgo anterior con la vinculación a una institución hospitalaria.

Los hallazgos ratifican lo que se documenta en la literatura con respecto a la mayoría de mujeres que deben asumir múltiples roles a la vez, incluido el de cuidadoras (33). De igual forma señalan la problemática de personas jóvenes con enfermedad crónica y discapacidad (3). De otra parte se ratifica que la mayor proporción de cuidadores asisten a sus familiares en el hogar (34). Por el contrario, este estudio muestra diferencias en el tiempo y en especial en la experiencia de los primeros 18 meses de cuidado con respecto a reportes anteriores $(7,10)$.

En cuanto a la habilidad de cuidado se ve con claridad que es homogénea en los dos grupos antes de iniciar la intervención y se hacen notorias algunas diferencias al finalizar el programa. Los dos grupos incrementaron el nivel del componente valor de la habilidad de cuidado. Sólo el grupo de estudio incrementó el nivel del componente conocimiento. Con respecto al componente paciencia se encontró una disminución en el grupo control y un incremento en el grupo de estudio. Al finalizar la intervención, la habilidad total y el componente paciencia tuvieron una diferencia estadísticamente significativa entre los grupos, siendo mejor el resultado del grupo de estudio. Hallazgos similares con programas para cuidadores han sido reportados en otras latitudes, sin embargo ninguno de estos se ha desarrollado alrededor del fomento de la habilidad del cuidado (11,35-37). 
Podría pensarse que el deterioro propio de la cronicidad que viven los receptores del cuidado afecta tanto la habilidad total de cuidar de sus cuidadores como cada uno de sus componentes y en tal sentido mantenerlos al mismo nivel puede significar un logro. No es pues sorprendente que tanto el componente valor como la paciencia del grupo control disminuyeran.

De otra parte, no se puede desconocer que los niveles de conocimiento son más elevados al inicio del estudio que los de los otros dos componentes, quizá porque la condición misma de cronicidad lleva a las personas a conocerse mejor con la experiencia. Esto en parte puede explicar la baja modificación en este componente entre los momentos inicial y final y el ligero incremento que esta característica tuvo en cada grupo.

Puede afirmarse con base en el estudio que fue más efectivo frente a la habilidad de cuidado el programa "Cuidando a los cuidadores" que las intervenciones tradicionales.

Puesto que las narrativas de quienes participaron en el programa señalan un cambio más significativo que el reflejado por los datos numéricos, puede pensarse que el CAI es útil para esta medición pero no suficientemente sensible frente al cambio en la habilidad para cuidar.

La presente investigación, con la evaluación positiva de un programa para el desarrollo de habilidades de cuidado dirigido a cuidadores familiares de personas con enfermedad crónica, genera un avance significativo que se suma a los reportados en la literatura, pero a diferencia de estos lo hace en América Latina. Los resultados aportan evidencia para recomendar el empleo del programa "Cuidando a los cuidadores" como una alternativa sencilla y viable para la región, que no implica altos costos y puede significar un cambio en el nivel de habilidad que los cuidadores requieren para asistir a un familiar que viva con enfermedad crónica.

El programa será replicado con enfermeros(as) que lideran la investigación y cualificación de esta práctica en los diferentes países y ciudades que se han unido a estos esfuerzos, para poder definir con los resultados de los mismos las necesidades sociales y de salud tendientes a mejorar la habilidad de cuidar de cuidadores de personas con enfermedad crónica. Son esfuerzos de esta naturaleza, sistematizados, metodológicamente soportados y colectivamente construidos los que la Universidad Pública está comprometida a mostrar como respaldo de su razón de ser cuando se tiene un norte en el fortalecimiento de la investigación y el desarrollo académico. 
Agradecimientos. A Colciencias, proyecto código 1101-04-13122, contrato No. 543 - 2002 y a la Facultad de Enfermería de la Universidad Nacional de Colombia, por su apoyo para el desarrollo del conocimiento para el cuidado a los cuidadores familiares de personas con enfermedad crónica.

\section{REFERENCIAS}

1. Sánchez B. El cuidado de la Salud de personas que viven situaciones de enfermedad crónica en América Latina. Actualizaciones en Enfermería 2002; 5 (1): 13-16.

2. Moreno ME, Náder A, López De MC. Relación entre las características de los cuidadores familiares de pacientes con discapacidad y la percepción de su estado de salud. Avances en enfermería 2004; (22):27-38.

3. Barrera L. La cronicidad y el cuidado familiar, un problema durante la niñez. Avances en enfermería 2004; (22)1:46-52.

4. Pinto N. La cronicidad y el cuidado familiar, un problema de todas las edades: los cuidadores de adultos. Avances en enfermería 2004; (22)1:53-59.

5. Sánchez B. La cronicidad y el cuidado familiar, un problema de todas las edades: los cuidadores de ancianos. Avances en enfermería 2004; (22)1:60-64.

6. Pinzón ML. Soporte social y afrontamiento a la enfermedad en adultos jóvenes sometidos a diálisis peritoneal ambulatoria. Avances en enfermería 2001; 19(2):47-53.

7. Mausbach BT, Coon DW, Depp C, Rabinowitz YG, Wilson E, Kraemer HC, et al. Ethnicity and Time to Institutionalization of Dementia Patients: A Comparison of Latina and Caucasian Female Family Caregivers. Journal of the American Geriatrics Society 2004; 52(7):1077-1084.

8. Álvarez BE. Comparación de la calidad de vida de cuidadores familiares de personas que viven con el VIH/Sida y reciben terapia antiretroviral, con la calidad de vida de cuidadores familiares de personas que viven con el VIH/Sida y no reciben terapia antiretroviral, del Instituto Nacional de Tórax y Hospital Mario Catarino Rivas. Avances en enfermería 2004; (22)2:618.

9. Merino S. Calidad de vida de los cuidadores familiares que cuidan niños en situación de enfermedad crónica. Avances en enfermería 2004; (22)1:47-53.

10. Lucke K, Coccia H, Goode J, Lucke J. Quality of life in spinal cord injured individuals and their caregivers during the initial 6 months following rehabilitation. Quality of Life Research 2004; 13(1):97-110.

11. Sörensen S, Pinquart M, Duberstein P. How Effective Are Interventions With Caregivers? An Updated Meta-Analysis. The Gerontologist 2002; 42(3); pp. 356-372.

12. Jeon YH, Brodaty H, Chesterson J. Respite care for caregivers and people with severe mental illness: literature review. Journal of Advanced Nursing 2005; 49(3):297-306. 32571005434 
13. Galvis CR, Pinzón ML, Romero E. Comparación entre la habilidad de cuidado de cuidadores de personas en situación de enfermedad crónica en Villavicencio Meta. Avances en enfermería 2004; (22)1:4- 26.

14. Barrera L, Pinto N, Sánchez B. Reflexiones sobre el cuidado a partir del programa "Cuidando a los cuidadores”. Aquichán 2005; 5(1):128-137.

15. Gannotti M, Kaplan LC, Handwerker WP, Groce NE. Cultural Influences on Health Care Use: Differences in Perceived Unmet Needs and Expectations of Providers by Latino and Euro-American Parents of Children with Special Health Care Needs. Journal of Developmental and Behavioral Pediatrics 2004; 25(3):156-165.

16. Alegria M, Canino G, Lai S, Ramirez R, Chavez L, Rusch D, Shrout PE. Understanding Caregivers Help-Seeking for Latino Children's Mental Health Care Use. Medical Care 2004; 42(5):447-455.

17. Svavarsdottir EK. Caring for a child with cancer: a longitudinal perspective. Journal of Advanced Nursing 2005; 50(2):153-161.

18. Shyu YI, Liang J, Wu CC, Su JY, Cheng HS. Chou SW, et al. A Pilot Investigation of the Short-Term Effects of an Interdisciplinary Intervention Program on Elderly Patients with Hip Fracture in Taiwan. Journal of the American Geriatrics Society 2005; 53(5):627-634.

19. Reever KE, Mathieu E, Dennis MP, Gitlin LN. Adult Day Services Plus: Augmenting Adult Day Centers With Systematic Care Management for Family Caregivers. Alzheimer's Care Quarterly 2004; 5(4):332-339.

20. Chang BL, Nitta S, Carter PA, Markham YK. Perceived Helpfulness of Telephone Calls: Providing Support for Caregivers of Family Members With Dementia. Journal of Gerontological Nursing 2004; 30(9):14-21.

21. Rotondi A, Sinkule J, Spring M. An Interactive Web-based Intervention for Persons With TBI and Their Families: Use and Evaluation by Female Significant Others. Journal of Head Trauma Rehabilitation. Focus on Clinical Research and Practice 2005; 20(2):173-185.

22. Glueckauf R, KettersonT. Telehealth. Interventions for Individuals With Chronic Illness: Research Review and Implications for Practice. Professional Psychology - Research \& Practice 2004; 35(6):615-627.

23. Nahikian M. Influential Factors of Caregiver Behavior at Mealtime: A Study of Twenty-Four Day Care Programs. Journal of the America Dietetic 1997; 97(5):505-509.

24. Sheehan N. The Caregiver Information Project: a mechanism to assist religious leaders to help family caregivers. The Gerontologist 1989; 29(5): 703-706.

25. Zarit S, Toseland R. Current and future direction in family caregiving research. The Gerontologist 1989; 29(4): 481-483.

26. Clark M, Lester J. The Effect of Video-Based Interventions on Self-Care. Western Journal of Nursing 2000; 22(8):895-911.

27. Burks K. A nursing practice model for chronic illness. Rehabilitation Nursing 1999; 24(5):197-200.

28. Hayes J. Respite for caregivers. A community-based model in a rural setting. Journal of Gerontolgical Nursing 1999; 25(1):22-26. 
29. Nkongho N. Working- Out Caregiving Systems in African American Families. Applied Nursing Research 1996; 9(3):108-114.

30. Newman MA. The pattern that connects. Advances in Nursing Science 2002; 24(3):1-7

31. Picard C, Jones D. Giving Voice to What We Know. August, 2004, Jones \& Bartlett Publishers, Inc. pp. 3-9.

32. Granger C, Greer D, Liset E, Coulombe J, O’Brien E. Measurement of Outcomes of Care for Stroke Patients. Stroke 1975; 6(1):34-41.

33. Ospina DE, Soto CP. Entre el rito y la rutina: el rol de cuidadora en las mujeres de Medellín. Investigación y educación en enfermería 2001; 19(2):58-65.

34. Sánchez B. La experiencia de ser cuidadora de una persona en situación de enfermedad crónica. Investigación y educación en enfermería 2001; 19(2):36-51.

35. Toseland RW, McCallion P, Smith T, Banks S. Supporting Caregivers of Frail Older Adults in an HMO Setting. American Journal of Orthopsychiatry 2004; 74(3):349-364.

36. Campbell AS. How was it for you? Families' experiences of receiving Behavioural Family Therapy. Journal of Psychiatric \& Mental Health Nursing 2004; 11(3):261-267

37. Mittelman MS, Roth DL, Coon DW, Haley WE. Sustained Benefit of Supportive Intervention for Depressive Symptoms in Caregivers of Patients With Alzheimer's Disease. American Journal of Psychiatry 2004; 161(5):850-856 